\title{
Interitem encoding and directed search in free recall
}

\author{
ROBERT M. HOGAN \\ Rockefeller University, New York, New York 10021
}

\begin{abstract}
The paper proposes a cognitive structure consistent with principles of encoding and a rule for its utilization in verbal recall. The encoding and utilization rules lead to phenomena similar to known serial position effects. A detailed analysis of overt rehearsal data and other experimental results are presented in support of the claim that encoding and search mechanisms characterized by these rules could be important factors in serial position effects of verbal recall. Similar rules might be helpful in dealing with other tasks which seem to mirror the utilization of a cognitive structure.
\end{abstract}

This paper is concerned with bridging the gap between certain well documented findings in verbal recall and a theoretical commitment common in cognitive and psycholinguistic research.

Current ideas about a range of verbal activities [including lexical decision tasks (Meyer, Schvaneveldt, \& Ruddy, 1972), semantic verification tasks (Rips, Shoben, \& Smith, 1973), analogical reasoning (Rips et al., 1973; Rumelhart \& Abrahamson, 1973), judgment of word similarity, generation of restricted associates, word guessing games, and free recall (each examined by Kintsch, Miller, \& Hogan, 1970)] appear to converge on the proposition that subjects make intelligent use of internal cognitive structure. The task which falls to the investigator is to develop a precise description of this structure and its utilization in given experimental situations.

While these tasks all illustrate the utilization of preexperimentally acquired cognitive structures, verbal recall research has tended to emphasize the importance of experimentally manipulable operations of encoding (though encoding operations themselves may be strongly influenced by preexperimental habits). What follows is an attempt to describe precisely the properties and utilization of a cognitive structure resulting from specific operations of encoding in the free recall situation.

The paper has four sections. In the first section, rules are proposed, determining a structure attributable to interitem encoding sequence and specifying how the structure is utilized during retrieval. The second section

Based on part of a doctoral dissertation submitted to the University of Colorado. The dissertation was supported by an NSF predoctoral fellowship and by NIMH Grant MH-15872 to W. Kintsch, while the present paper was written during the author's tenure as postdoctoral fellow at The Rockefeller University, supported by PHS Grants GM 01789 and GM 16735. I received the encouragement and advice of Walter Kintsch, thesis advisor. The paper has also benefited from suggestions and criticism by Edward J. Crothers, W. K. Estes, Rachel J. Falmagne, Mary M. Hogan, and Dirk Vorberg. Clarity of exposition was improved on the basis of comments by two unknown reviewers. An abbreviated version of this paper was presented at the Mathematical Psyehology meetings, Montreal, August 1973. demonstrates that a process characterized by the proposed rules could contribute to known serial position effects in verbal recall, i.e., that serial position effects might evolve from specifics of encoded structure. In the third section, the rules are used to derive quantitative expressions for serial position phenomena as functions of the pattern of attention allocation mirrored by the encoded structure. The fourth section reports an experiment that used sequential information available from overt rehearsal data to (a) directly test expressions derived in the previous section, (b) examine several alternative rules mapping patterns of encoding to cognitive structures, and (c) investigate a sequential encoding interpretation for results of Rundus (1971) manifesting a high correlation between rehearsal frequency and retention.

\section{THE ENCODED STRUCTURE AND ITS UTILIZATION}

To do justice to the ubiquity of organizational strategies in verbal recall, theories cannot be limited to predicting the memory status of an "average item" but must be sensitive to structural properties of "accessible memory units" (Tulving, 1967). Accessible memory units effect "search paths" (Mandler, 1968) between arbitrary items, so an adequate description of verbal recall must be concerned with the likelihoods of all possible item-to-item transitions during retrieval and with how these likelihoods are influenced by processes of encoding.

Consider the following simple hypothesis: During retrieval the likelihood of any transition from list item $i$ is given by the relative frequency of the corresponding transition during encoding. Suppose that, by means of the overt rehearsal procedure or more indirectly, ${ }^{1}$ it has been possible to evaluate the frequencies of all transitions between representations of list items during encoding. For an n-item list, with ordered indices corresponding to serial positions during presentation, this would give an $\mathrm{n}$ by $\mathrm{n}$ frequency matrix, 


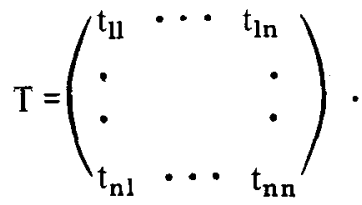

The corresponding $n$ by $n$ probability matrix

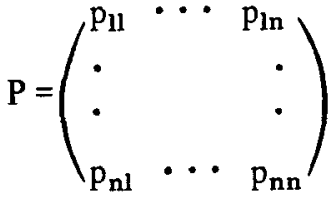

may be obtained by normalizing each row of $\mathrm{T}$ (i.e., dividing every element of each row by its row sum). The matrix $\mathrm{P}$ will be referred to as the encoded structure relevant to serial position effects in verbal recall.

The matrix $\mathbf{P}$ contains the relative frequencies of all transitions during encoding; the information in $\mathbf{P}$, therefore, determines the relative likelihood of any transition during retrieval. Given that the item from serial position i (call it item i) has just been "drawn," the probability of next choosing item $\mathrm{j}$ is given by element $\mathrm{p}_{\mathrm{ij}}$ of matrix $\mathrm{P}$.

The hypothesis says nothing about where a given retrieval sequence begins. If a certain retrieval sequence begins with item $\mathrm{k}$, the hypothesis does, however, specify the likelihood of drawing the item from any serial position at any later point in the sequence, conditional upon search initiating at point $k$. For practical reasons, most of what follows is restricted to characterizing serial position data averaged over subjects and lists. Using the symbol $\ddot{\vec{\pi}}$ to refer to a normalized frequency distribution of initiation points over subjects and lists and evaluating $P$ from encoding of the same subjects on the same lists, the hypothesis specifies that the mean probability of retrieving an item from any of the $n$ serial positions on the $q^{\text {th }}$ draw $\left(q+1^{\text {th }}\right.$ item $)$ of a verbal recall retrieval sequence will be given by the $n$ vector

$$
\overrightarrow{\mathrm{R}}_{\mathrm{q}}=\vec{\pi} \mathrm{Pq} .
$$

This is the rule for utilization of $P$.

Consider briefly the type of matrix likely to result from patterns of interitem encoding. For list items presented sequentially, the predominant tendency is for attention to go from prior items to the one presented, with perhaps a few repetitions, then back to the previous item and others from the previous rehearsal set (Rundus, 1971). This pattern of distributing attention should, in general, lead to a regular matrix P (Kemeny \& Snell, 1961), with nonzero likelihood of a path from any item to any other. In what follows, it will be assumed that the encoded structure $\mathbf{P}$ is a regular probability matrix.
If $P$ is regular, the probability vector $\vec{\pi} P a$ of Equation 1 has a limiting distribution over the $n$ serial positions defined by

$$
\bar{\delta}=\lim _{q \rightarrow \infty} \vec{\pi} \mathrm{Pq} .
$$

This distribution may be found by solving

$$
\bar{\delta}(\mathrm{P}-\mathrm{I})=0,
$$

where $I$ is the identity matrix of order $n$, or approximated to any degree of accuracy by evaluating $\vec{\pi} \mathrm{Pq}$ for a sufficiently large integer $\mathrm{q}$. The vector $\vec{\delta}$ will be called the equilibrium distribution for the search process governed by $\vec{\pi}$ and $\mathrm{P}$. This vector has the important property of not depending upon q or upon $\vec{\pi}$; $\vec{\delta}$ may be thought of as "structural," in the sense that it is totally determined by the previous pattern of encoding.

\section{STRUCTURAL AND TRANSIENT COMPONENTS OF MEMORY SEARCH}

Waugh and Norman (1965) proposed that R(i), the probability of recalling an item followed by $i$ subsequent items; $P(i)$, the probability of the item's presence in primary memory; and $S(i)$, the probability of its presence in secondary memory; are related by

$$
R(i)=P(i)+S(i)-P(i) S(i) .
$$

$\mathrm{P}(\mathrm{i})$ is characterized by transience; this property is made explicit in the assumption that $\mathrm{P}(\mathrm{i})$ is a monotonically decreasing function of $i$ with $\lim _{i \rightarrow \infty} P(i)=0$. $S(i)$, on the other hand, has permanence; Waugh and Norman restricted their discussion to cases where $S(i)$ is greater than zero and independent of $i$.

Plausible as this decomposition appears, its use has been limited in two respects: First, the quantities involved have been estimated from serial position data rather than predicted on the basis of specific assumptions about encoding and retrieval. Second, for an item presented in serial position $j$ and retrieved on draw $\mathrm{q}$, an expression for $\mathrm{R}(\mathrm{i})$ must be the limiting case of a potentially more informative expression for $R(j, q)$. Explicit dependence upon $\mathrm{j}$ may not have been necessary to summarize data considered by Waugh and Norman; it would appear to be mandatory in dealing with phenomena of primacy (e.g., Bruce \& Papay, 1970) or negative recency (Craik, 1970).

Equation 1 is subject to neither of the above limitations, as it gives an expression for $R(j, q)$ based on specific assumptions about encoding and retrieval. The serial position information contained in $R(j, q)=(\vec{\pi} P q)_{j}$ is illustrated in Figure 1. The figure shows distributions $\overrightarrow{\bar{\pi}} \mathrm{P}^{\mathrm{q}}$ for $\mathrm{q}=0,1,2,3$, and a large integer $\mathrm{n}$, each 


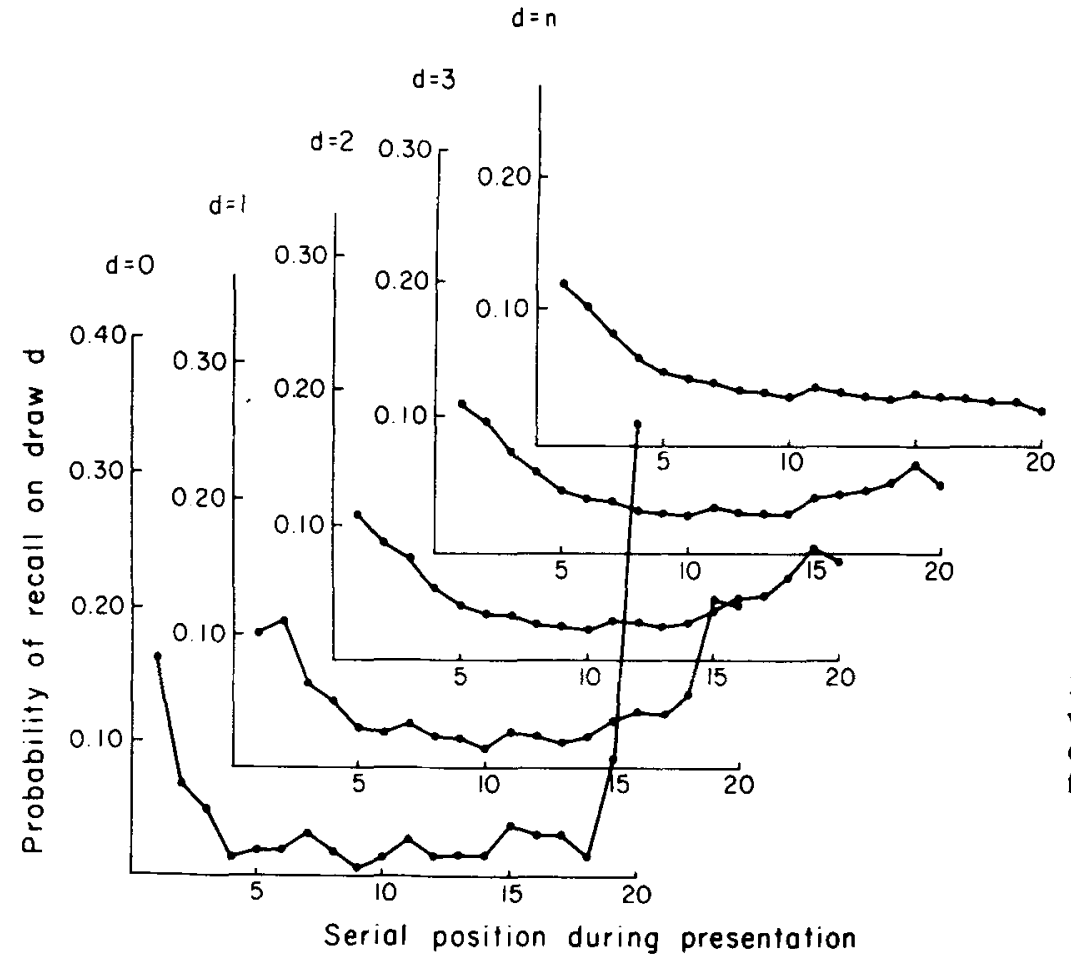

Figure 1. Predicted serial position curves, $\vec{\pi} \mathbf{P q}$, for the individual draws $\mathrm{q}=0,1,2$, 3 , and large integer $n$. The vector $\vec{\pi}$ used was $\bar{\pi}_{\text {RECALL (see Table } 2 \text { ) and } P \text { was }}$ evaluated in accordance with the model from overt rehearsal data.

evaluated using collected search initiation point data and/or overt rehearsal data. The matrix $\mathbf{P}$ was computed from patterns of rehearsal according to the rules stated earlier; the various distributions were then evaluated by means of a computer program.

The first distribution shown in Figure 1 is $\vec{\pi}$, which corresponds to $q=0$. This distribution was evaluated by the proportions, from each of 20 serial positions, of items which occurred first in response sequences. Notice that all other distributions, including the few shown in Figure 1, are predicted on the basis of specifics of encoding as mirrored by patterns of rehearsal. No other model concerned with verbal recall has characterized the retrieval process in this detail.

Notice the distribution at $q=n$. This distribution approximates the limiting vector $\bar{\delta}$ of the search process governed by $\vec{\pi} \mathrm{Pa}$. The limiting vector thus possesses a strong primacy component, a central plateau, and negative recency.

The predicted distributions in Figure 1, which are qualitatively consistent with phenomena of primacy and negative recency, also appear to reflect properties that have come to be associated with primary and secondary memory. There seem to be "transient" components to these distributions which cause earlier ones to deviate systematically from $\bar{\delta}$ and which become relatively less important as position in the response sequence increases.

The pattern of decreasing transient components is not an accidental result of the particular matrix $P$ used here but can be shown to follow from models of the present type for a wide class of matrices, under reasonable assumptions relating the equilibrium distribution $\bar{\delta}$ to the likelihood of retrieval from secondary memory. However, the detailed argument is relatively complex and a digression from what follows. It will be presented at a later time.

An additional point of interest with regard to the transient components in Figure 1: If there is any tendency (as shown by $\vec{\pi}$ of Figure 1) for response sequences to start elsewhere than at list's end, then the transient components predicted by this model are not restricted to the most recent serial positions. This prediction is easily tested and forms the basis for an experimental manipulation reported later in the paper.

It should be noted that the structural and transient components to which reference has been made are components of the probability of recall due to an assumed interitem memory search; they stand noncommittal about the number or nature of subsidiary mechanisms of storage.

\section{SERIAL POSITION CURVES FOR ACCURACY AND ORDER OF FREE RECALL}

The preceding section shows how phenomena that have come to be associated with primary and secondary memory could evolve from the sequential memory search characteristic of verbal recall. But the practical usefulness of this framework remains to be determined. Even if the model is wholly correct in describing a process which occurs in verbal recall, it could happen that other processes are more important, with the 
"memory search component" of serial position curves being small. To investigate this possibility, quantitative predictions are needed for the serial position curve components directly attributable to interitem search.

By Equation 1 it is known that on the $\mathrm{q}^{\text {th }}$ draw of a retrieval sequence, the occurrence of the item presented in serial position $\mathrm{j}$ has mean probability

$$
R(j, q)=(\vec{\pi} P q)_{j}
$$

This expression describes a search process of "sampling with replacement," so the possibility exists that, when item $j$ is drawn, it has already occurred on a prior draw. Overt repetitions are relatively rare in most verbal recall tasks, suggesting that subjects edit their reports of "repetitions" quite accurately, but this view is still preferable to its altemative, "drawing without replacement."

It is necessary to isolate the likelihoods of the mutually exclusive events, first occurrence or repetition, of item $\mathrm{j}$ on draw q. This is done in

$$
\begin{aligned}
& R(j, q)=R^{\prime}(j, q)+{ }_{n=0}^{q} \bar{\Sigma}^{1}\left(P^{q-n}\right)_{j j} R^{\prime}(j, n), q \geqslant 1 \\
& R(j, 0)=R^{\prime}(j, 0)=\pi_{j} .
\end{aligned}
$$

Equation 2 breaks the probability of occurrence of item $\mathrm{j}$ on draw $\mathrm{q}$ down into its probability of occurring for the first time on draw $\mathrm{q}\left[\mathrm{R}^{\prime}(\mathrm{j}, \mathrm{q})\right]$, plus its probability of occurring for the second or any subsequent time on draw q.

The correction factor for repetitions is a sum, with one term corresponding to each possible draw at which $j$ might occur for the first time, given that it occurs again on draw q. The correction factor may, in general, be evaluated by a recursive process from knowledge of $\vec{\pi}$ and P. A particularly simple example of the computations involved is given in Appendix A. For higher draws and for matrices of larger order, it becomes necessary, as with most matrix multiplications, to do the calculations by computer.

While the appearance of Equation 2 is superficially intimidating, this added complexity has its reward. The two terms constitute separate and distinct predictions for "first recall" and "repetition" serial position phenomena, each specified with the detail illustrated in Figure 1.

It would be possible to test the predictions for each draw separately but, as a preliminary test of the model's feasibility, certain approximations will be made to estimate the more usual serial position statistics. To the extent that these approximations characterize the data, more fine-grained analyses may be undertaken.

The probability of ultimately recalling the item from serial position $j$ during an entire test interval may be obtained by summing $R^{\prime}(j, \dot{q})$ appropriately over $q$. The probability of an item being recalled during d draws is equal to the sum of the item's probabilities of being recalled for the first time on draws $0,1, \ldots, d$. By estimating the mean number of draws, $\bar{d}$, during a test interval, one could employ the following approximation for the likelihood of item $j$ occurring within the test interval:

$$
P(\text { Recall })_{j}=\sum_{q=0}^{\bar{d}} R^{\prime}(j, q) .
$$

In making this approximation, individual differences and list differences are ignored but this is the common limitation of current memory models. Equation 3 will permit a preliminary judgment about the promise of this approach.

Order data in verbal recall has been summarized (Bousfield, Whitmarsh, \& Esterson, 1958; Deese \& Kaufman, 1957) by plots of mean recall position within the retrieval sequence as a function of serial position during presentation. It is possible to develop an approximate expression for the form of this type of serial position curve, as well, by an appropriately weighted summation.

The statistic used in the preceding articles was computed from sequences of correct responses, so the present theoretical computation must be conditional upon successful recall. Again using $\bar{d}$ to approximate the influence of test interval, the distribution $R^{\prime}(j, q)$ (which for item $j$ is a probability distribution over $q$, $q=0,1, \ldots)$ can be conditionalized upon the occurrence of item $j$ for the first time within a sequence of $\bar{d}$ draws. This is done for item $j$ by dividing $R^{\prime}(j, q)$ by $P(\text { recall })_{j}$. For each item $j$, this operation defines a new function

$$
R^{\prime \prime}(j, q)=\frac{R^{\prime}(j, q)}{P(\text { recall })_{j}}
$$

which corresponds to the item's probability of being retrieved for the first time on draw $q$, given that it is successfully retrieved during $\bar{d}$ draws.

The function $R^{\prime \prime}(j, q)$ is a probability distribution over the range $q=0,1, \ldots \bar{d}$, so it is possible to compute $X_{j}$, the predicted mean first recall position for item $\mathrm{j}$. The expected value of the random variable corresponding to first recall position for item $j$ is

$$
X_{j}=1+\sum_{q=0}^{\bar{d}} q R^{\prime \prime}(j, q) \text {. }
$$

Again this summation will be less precise than one which takes into account individual differences and list differences. Equation 4 will nevertheless be utilized to judge the promise of this approach.

\section{EXPERIMENT}

This was a modified free recall study, designed to investigate the class of interitem encoding and search models and, specifically, to test Equations 2, 3, and 4 . 
Equations 3 and 4 predict mean recall accuracy and order as functions of serial position from knowledge of $\mathrm{P}, \overline{\bar{\pi}}$, and $\overline{\mathrm{d}}$. The matrix $\mathrm{P}$ would be evaluated from recorded overt rehearsal patterns. The mean number of draws, $\bar{d}$, would be estimated by mean response sequence length, as subjects were required to recall without editing repetitions. The vector $\vec{\pi}$ would correspond to the distribution of items recalled first in each experimental condition.

Known properties of recall order and accuracy might be at least partially attributable to interitem memory search. However, additional sources of information (acoustic cues, articulatory cues, or "encoded" short-term information) may influence retention as measured by verbal recall. Stores associated with these sources have uniformly been suggested by "recency" phenomena. In this experiment, an effort was made to influence the transient component due to memory search in a way that would separate it from other sources. If, as suggested by the current model, a transient component of "recency" effects is actually determined by where the search begins, it should be possible to move this component elsewhere on the serial position curve by influencing $\vec{\pi}$.

To this end, experimental conditions were designed to differ only in their corresponding vectors $\pi$. The vectors would be influenced solely by the nature of a nonpredictable postlist cue to avoid influences of "control processes" during encoding. Conditions would also minimize the salience of the usual acoustic and articulatory cues. Under such conditions, it was hoped that the initial vector $\overrightarrow{\vec{\pi}}$ would be influenced by the postlist cue in a way discussed further in the Method section. Whatever the resulting $\vec{\pi} \mathrm{s}$, the model requires that predictable portions of the "recency" effect be transferred to different locations on the serial position curve.

It was hoped that the components transferred would be substantial. To test whether the transferred components were transient, like the recency component reported by Craik (1970), a final recall task was introduced at the experiment's conclusion. If postlist cues had been effective in changing the form of immediate recall serial position curves, then final recall serial position curves would attest to whether the changes were temporary or lasting.

\section{Method}

Subjects. The subjects were 40 students at the University of Colorado who were either paid or participated as a course requirement.

Materials. Items were one-syllable words, three or four letters long, with final consonants $b, d, g, k, 1, n, p, r$, or $t$. One hundred and eighty of the words were chosen and randomly assigned to nine 20-word lists. A given word remained part of the same list; within lists, items were assigned quasirandomly so that each word appeared equally often in each serial position for each experimental condition.
Procedure. Lists were shown on a Stowe memory drum within a $10 \times 10 \mathrm{ft}$ soundproof cubicle. The experimenter and the subject were separated by a pegboard partition. Subjects participated individually. Each experienced nine 20-word lists, presented at the rate of $5 \mathrm{sec} /$ item. Every list was immediately followed by a spoken free recall test of $1-m i n$ duration. The signal to commence recall was the presentation of a red capitalized word which the subject was required to pronounce. Subjects were instructed to rehearse aloud by pronouncing members of the current list. This rehearsal was tape recorded. Subjects were also instructed not to edit their responses during recall (i.e., to say the same word twice if they thought of it twice). Following the test for List 9 , the subjects participated in a 1-min arithmetic task (paced backward counting). They were then allowed $5 \mathrm{~min}$ for spoken free recall of items from all lists.

Design. The first list shown was considered a practice list. Data for Condition RECALL comes from four lists that were immediately followed by the prefix cue "RECALL." Data for Condition 6 comes from two lists that were immediately followed by the sixth word of the list as prefix cue. Data for Condition 13 comes from the two remaining lists, which were followed by the 13th list word as prefix cue. Conditions were randomly assigned to list positions 2 through 9 , subject to the constraint of uniform distribution over positions.

The irrelevance of the prefix word was stressed in instructions; nevertheless, it was expected that memory searches cued by list items would tend to start closer to the item's position, since irrelevant information cannot be completely ignored.

\section{Results and Discussion}

Rehearsal Data: T and $\bar{\delta}$. The transition frequency matrix $\mathrm{T}$ computed from rehearsal data is presented as Table 1. This table summarizes rehearsal frequency and transition information obtained from all subjects and conditions.

The matrix $T$ has no cell with zero frequency, so its normalized stochastic matrix, $\mathrm{P}$, is obviously regular. The same conclusion would hold even if some of the lower frequencies were zero, because every serial position shows strong tendencies for repetitions and transitions to adjacent positions.

Since $\mathbf{P}$ is regular, it possesses a limiting vector $\vec{\delta}$. The vector $\vec{\delta}$ shows primacy, a level central plateau, and negative recency. It is almost exactly proportional to the vector of column sums in Table 1, i.e., to the corresponding vector of rehearsal frequencies.

Rundus (1971) has reported suggestive correlations between rehearsal frequency and accuracy of free recall. The fact that in this study rehearsal frequency was proportional to $\vec{\delta}$ suggests that interitem encoding could underlie Rundus's results. This possibility is further investigated in Appendix B, which isolates a class of frequency matrices for which the proportionality between rehearsal frequencies and $\vec{\delta}$ must occur.

Influence of Condition on $\vec{\pi}$. The initial vector $\vec{\pi}$ was determined for each condition by the distribution over serial positions of the initial item recalled for each subject-list combination. Table 2 indicates that the vectors $\vec{\pi}_{\text {RECALL}}, \quad \vec{\pi}_{6}$, and $\vec{\pi}_{13}$ did differ systematically. Condition 6 was effective in moving initiation points from list's end to Serial Positions 1 
Table 1

Rehearsal Transition Frequency Matrix $T$

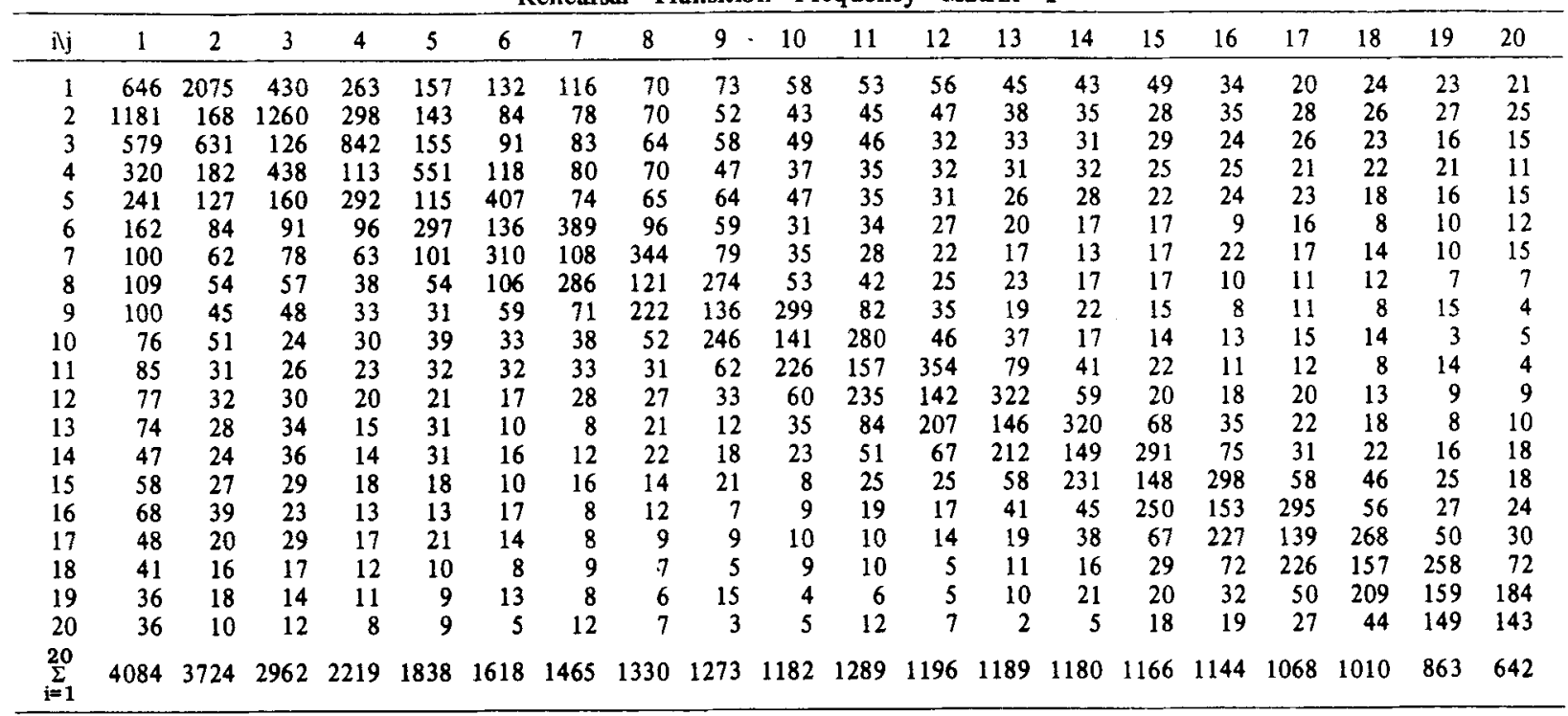

through 5. Condition 13 was equally proficient in removing initiation points from Positions 19 and 20 but not as selective in where the points were redistributed.

Although the vectors $\vec{\pi}_{\text {RECALL }}, \vec{\pi}_{6}$, and $\vec{\pi}_{13}$ differ, this was by no means the strongest possible manipulation of $\vec{\pi}$. In fact, the final serial position remained the most likely initiation point in each of the three conditions.

Mean Number of Draws, $\overline{\mathbf{d}}$. To the extent that subjects avoided editing in compliance with the instructions, the number of draws during immediate retrieval was an observable quantity. The mean observed number of draws (items recalled, including repetitions, minus 1) was $12.0,12.2$, and 11.8 for Conditions 6,13 , and RECALL, respectively. The integer 12 was therefore substituted for $\bar{d}$ in computations using Equations 3 and 4.

Serial Position Data. The observed proportions of correct immediate recalls are summarized for condition and serial position block in Table $3 \mathrm{~A}$. The relatively small recency component under all conditions is consistent with the present attempt to minimize sensory cues and may be attributable to a number of factors, including the use of spoken rather than written recall (Craik, 1969) and the presence of a spoken response prefix (Crowder, 1970).

In spite of this small recency component and the relatively weak influence of experimental conditions, the trading relation visible in Table $3 \mathrm{~A}$ is statistically reliable. A repeated measures analysis of variance performed on the number of correct responses for each subject-condition combination in Serial Positions 1-5, $8-12$, and 16-20 showed no effect of condition $[F(2,78)<1]$ but a reliable Condition by Serial Position interaction $[\mathrm{F}(4,156)=3.43, \mathrm{p}<.01]$. Planned comparisons within separate blocks of serial positions showed the following: In Positions 16-20 the proportion correct in Condition RECALL was greater than the mean proportion correct in Conditions 6 and 13 $[F(1,78)=10.6, p<.01]$, while Conditions 6 and 13 did not differ $[F(1,78)<1]$. In Positions $1-5$, Condition 6 was superior to the weighted average of the

Table 2

Frequency of First Word in Immediate Recall as a Function of Condition and Serial Position

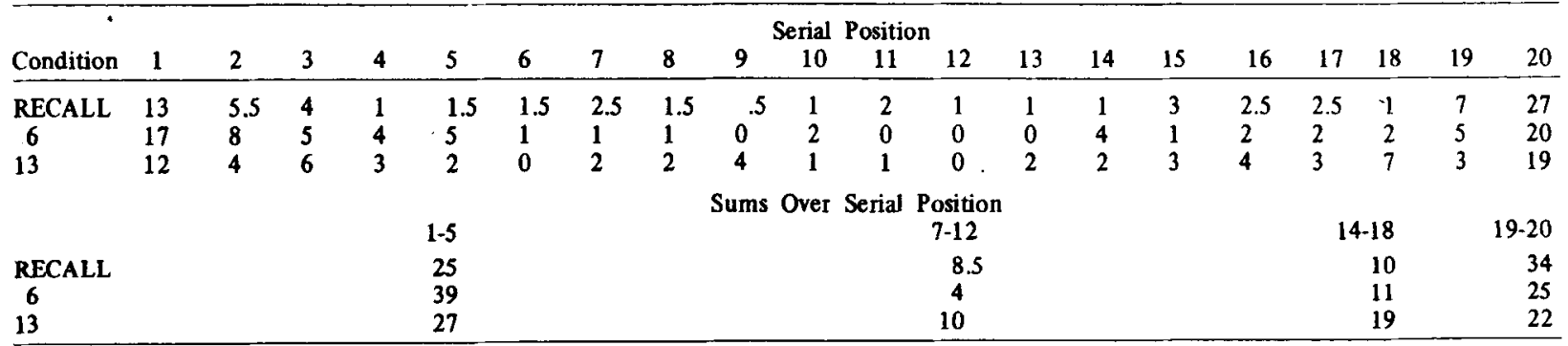

Note-Frequencies in Condition RECALL are divided by 2 for ease of comparison. The initial probability vectors were computed by dividing each of the entries by 80 . 
two other conditions $[F(1,78)=4.36, p<.05]$, while the others did not differ $[F(1,78)<1]$. A nonsignificant superiority for Condition 13 was observed over Positions 8.12 $[\mathrm{F}(1,78)=1.90, \mathrm{p}>.10]$, while the other two conditions did not differ $[F(1,78)<1]$. The ineffectuality of Condition 13 in transferring a component to central positions may be due to the lesser selectivity of this condition's influence on the distribution of search initiation points.

Concerning whether the early superiority for Condition 6 was transient or lasting, analysis of final recall data indicates that it was as fleeting as the recency superiority for Condition RECALL. This observation is supported by an analysis of variance, identical to the previous one, applied to final recall data. Unlike immediate recall, the final recall data showed no effect of Condition by Serial Position, $\mathrm{F}<1$; final recall of items from Condition 6 over Positions $1-5$ was actually slightly worse than from either Condition 13 or Condition RECALL. Immediate recall was about $60 \%$ and final recall about $40 \%$ over Positions 1.5 , so there is no reason to believe that the absence of a Condition 6 advantage in final recall was due to final recall being a less sensitive measure of retention.

These results give some support to the existence of a transient component of serial position curves, governed not by recency but by search initiation point. The size of the "transferred" component was not large. However, Cohen (1970), using postlist cues for free or ordered recall, also found differences over early serial positions which disappeared in final recall.

The Amount Transferred. The primary object of the present study was not to transfer the greatest possible "recency" component; it was to test whether the components transferred in a free recall situation were of the size predicted for essentially arbitrary $\vec{\pi}$ s. The small but reliable component transferred in Condition 6 and the absence of a visible transferred component in Condition 13 might stand totally consistent with Equation 3. Comparison of predicted and observed values in Table $3 \mathrm{~A}$ affirms that this was the case.

Immediate Recall Accuracy: Test of Equation 3. Panels $\mathrm{A}, \mathrm{B}$, and $\mathrm{C}$ of Figure 2 compare the observed serial position curve for each condition to that component of the curve predicted by Equation 3. The predicted values were computed using the pooled rehearsal transition frequency matrix, $\overline{\mathrm{d}}=12$, and $\vec{\pi}=$ $\vec{\pi}_{6}, \vec{\pi}_{13}$, or $\vec{\pi}_{\mathrm{RECALL}}$, respectively. It is evident that the model characterizes primacy and plateau regions in all conditions quite well, with systematic disagreement only over later serial positions. The observed values in Table $3 \mathrm{~A}$ deviate from the predicted values only at Serial Positions 14 through 20; over these positions, observed values are consistently greater than predicted. The deviations might reflect acoustic/articulatory (or other short-term) information, since there is no reason to believe that this source was completely eliminated.

The original hypothesis about interitem encoding and search, in conjunction with the approximation reflected in Equation 3, gives an adequate account over the first 13 serial positions in each condition (see Table 3A); while predictions are consistently too low over the final seven positions, there is some evidence to suggest that "transient" results of a search process consistent with the model can account for a significant portion of recency effects.

Repetitions. The "unedited" recall task provides additional data which presumably reflect memory search. In this experiment, repetitions (items recalled for

Table 3

(A) Mean Proportion of Correct First Recalls and Standard Errors of the Mean Proportion for Condition and Serial Position Block, With Corresponding Predicted Proportions in Parentheses

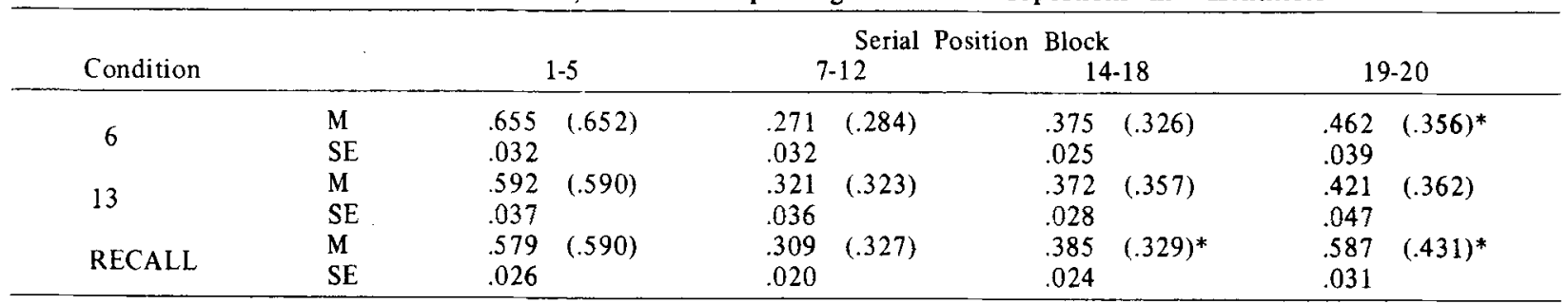

(B) Mean Frequency of Repetitions Per List and Standard Error of the Mean Frequency for Condition and Serial Position Block, With Corresponding Predicted Frequencies in Parentheses

\begin{tabular}{|c|c|c|c|c|c|c|c|c|c|}
\hline 6 & $\begin{array}{l}\mathrm{M} \\
\mathrm{SE}\end{array}$ & $\begin{array}{l}.405 \\
.059\end{array}$ & $(.533) \dagger$ & $\begin{array}{l}.121 \\
.027\end{array}$ & $(.112)$ & $\begin{array}{l}.147 \\
.027\end{array}$ & $(.176)$ & $\begin{array}{l}.194 \\
.050\end{array}$ & $(.253)$ \\
\hline 13 & $\begin{array}{l}\mathrm{M} \\
\mathrm{SE}\end{array}$ & $\begin{array}{l}.370 \\
.066\end{array}$ & $(.451)$ & $\begin{array}{l}.185 \\
.039\end{array}$ & $(.134)$ & $\begin{array}{r}.150 \\
029\end{array}$ & $(.215) \dagger$ & $\begin{array}{r}.156 \\
032\end{array}$ & $(.256) \dagger$ \\
\hline RECALL & $\begin{array}{l}\mathrm{M} \\
\mathrm{SE}\end{array}$ & $\begin{array}{l}.326 \\
.046\end{array}$ & $(.449) \dagger$ & $\begin{array}{l}.150 \\
.022\end{array}$ & $(.134)$ & $\begin{array}{l}.172 \\
.030\end{array}$ & $(.172)$ & $\begin{array}{l}.181 \\
.038\end{array}$ & $(.344) \div$ \\
\hline
\end{tabular}

* Observed exceeds predicted by more than two standard errors.

tPredicted exceeds observed by more than two standard errors. 


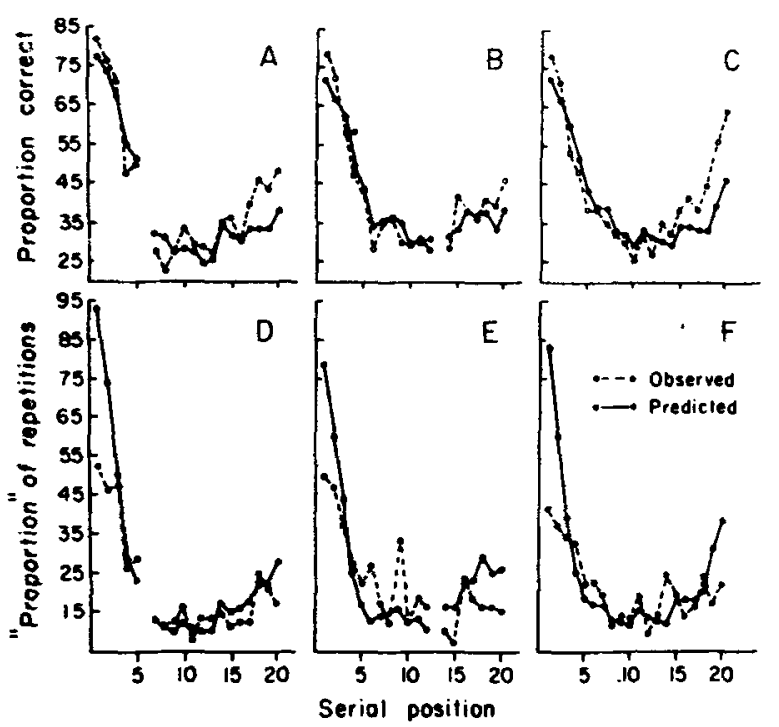

Figure 2. Observed and predicted proportions of recall (Panels $A, B$, and $C$ ) and observed and predicted frequencies of repetition per subject-list combination (Panels D, E, and F) for Conditions 6, 13, and RECALL.

the second, or subsequent, time) accounted for nearly $40 \%$ of all words reported.

If Equation 2 accurately separates "first recalls" from "repetitions" during retrieval, then by analogy with the argument leading up to Equation 3, an approximation to the expected "serial position curve" for repetitions may be obtained by appropriately summing the correction factor. Unfortunately, a fair test of this prediction would require total compliance with instructions; the distribution of observed repetitions could deviate markedly from the predicted distribution if subjects were reluctant to report items previously retrieved. Some conclusions might, nevertheless, be possible. Observations which reliably exceed predicted values would point to inadequacies in the model as stated. Observations which fall reliably below predictions would yield no strong conclusions but might reflect an editing process. Observations which substantially agree with predictions would clearly support the model.

Panels D, E, and F of Figure 2 compare observed and predicted likelihoods of repetition over serial position in each of the three conditions. The predicted likelihoods were computed by summing the repetition factor in Equation 2 from $q=0$ to $q=12$. These predicted curves differ from predicted "first recall" curves in that primacy is steeper and the central plateau appreciably lower. The panels show and Table 3B substantiates that the approximation gives a good account of repetition frequencies over central serial positions but that predictions exceed observations at the extremes.

Interpretation is difficult over Positions 14 to 20 because first recall data appeared to implicate a recency mechanism other than that characterized by the present model. The approximation gives a credible account over Positions 3 to 13, thereby supporting the model. An editing mechanism may have influenced the report of some items, particularly those presented in Positions 1 and 2. This final statement seems consistent with the pattern of results, including (a) the accurate predictions for first recall curves but excessive predictions for repetitions, (b) the predicted shape of repetition curves, which suggests that first presented items would be drawn with annoying frequency, and (c) more detailed analyses over individual subjects, which show large individual differences in number of reported repetitions.

Immediate Recall Order: Test of Equation 4. The panels of Figure 3 show mean position of first recall for items in the recall sequence as a function of condition and serial position of presentation. Recent items tended to be recalled first, followed by items presented early, and then middle items. The $U$-shaped nature of the curve is consistent with well established results (Bousfield, Whitmarsh, \& Esterson, 1958; Deese \& Kaufman, 1957; Rundus, 1971). Condition 6 resulted in a decrease of mean recall position for early items, but.standard errors were larger here relative to observed means.

Also visible in Figure 3 are comparable curves, computed from Equation 4, with $P$ equal to the pooled rehearsal probability matrix, $\overline{\mathrm{d}}=12$, and $\vec{\pi}=\bar{\pi}_{6}$, $\vec{\pi}_{13}$, or $\vec{\pi}_{\text {RECALL}}$, respectively. The predicted curves possess the same gross characteristics, with last items recalled first and intermediate ones last.

Although predicted and observed curves intersect, their shapes differ systematically. The three panels agree in showing that observed recall positions were lower than expected for early serial positions but greater than expected in late central positions. An important factor contributing to these deviations is the fact that late central items were recalled in disproportionate numbers from sequences of length greater than 12 and early items from sequences of length less than 12. A partition of long and short response sequences supports this interpretation, with early and late deviations clearly separable.
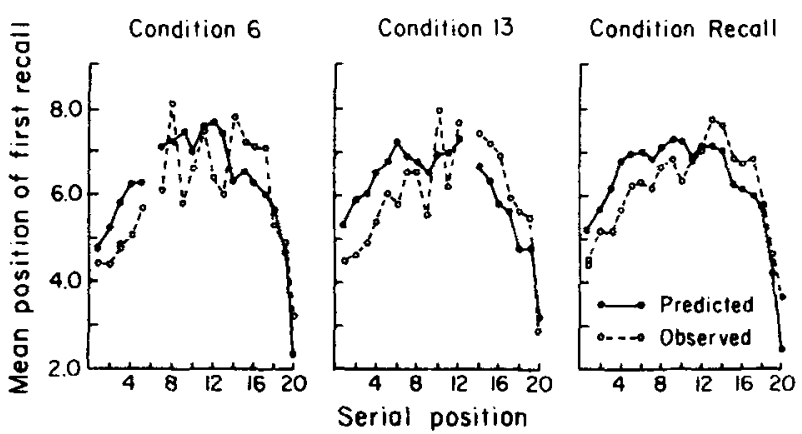

Figure 3. Observed and predicted mean positions of first recall as functions of serial position and condition. 
Equation 4, therefore, gives a generally accurate description of order data, but one in which systematic deviations are directly attributable to a limitation of the approximation procedure, which ignores individual differences in number of draws.

Conclusions. The approximation procedure introduced to estimate predicted components of the serial position curves for accuracy, order, and repetitions yields the following conclusions:

(1) The hypothesis, that probability of transition from $i$ to $j$ during retrieval is given by the relative frequency of the corresponding transition during encoding, implies that a substantial component of serial position curves in verbal recall may be attributable to memory search operations during retrieval, governed by a specifiable cognitive structure.

(2) To the extent that the approximations used are valid, this hypothesis permits accurate predictions for cumulative first recall and repetition accuracy curves, with the following exceptions: (a) The recency effect may partially reflect a transient component due to memory search but evidently requires an additional factor, perhaps sensory cues and/or a "recency" store. (b) Repetitions data may reflect a process of editing as well.

(3) Predictions for order of recall show systematic deviations which may be traced directly to an inadequacy of the approximation procedure, its insensitivity to individual and list differences. Further research with the model could clarify the extent to which these factors are important in particular experimental situations.

Generally, it appears that the results are sufficiently promising to merit further investigation of interitem encoding and search processes as potential mechanisms of verbal recall.

However, more direct tests of the hypothesis should be made under conditions where there is no "recency" factor, using first recall data which presumably shows no "editing." It would also be preferable for predictions to depend only on $\vec{\pi}$ and $\mathrm{P}$, with no approximation for number of draws. Tests reported in the next section meet these requirements.

Serial Position Curves for Individual Draws. The "first recall" serial position curves were examined for each condition at Draw 1 and Draw 2 (see Equation 2). Draws 1 and 2 should be maximally sensitive to the variable of condition. Each subject on every list made at least two draws, so there would be no problem of individual or list differences. Predictions were based solely on $\vec{\pi}$ and P. Only Serial Positions 1 to 13 were considered; prior analysis and other research suggest that these positions reflect no recency mechanism.

Predicted and observed proportions for each condition are presented in Table 4. A computed $\chi^{2}$ statistic for goodness of fit shows $\chi^{2}(70)=63.1$, $\mathrm{p}>.50 .^{2}$ One can also obtain some measure of how sensitively the model reflects individual draws by computing goodness of fit over the same data but with the predictions for Draws 1 and 2 switched, $\chi^{2}(70)=121.3, p<.01$. Evidently the good fit is not an inevitable consequence of using rehearsal data and depends on the observed serial position curves being more similar to the predicted serial position curves than they are to each other.

Individual Subjects and Lists. It would be possible to generate quantitative predictions by applying Equations 3 and 4 to individual rehearsal protocols, but such predictions are subject to all of the complications isolated by prior analysis of averaged data and several new problems unique to the individual case. The following procedure is less ambitious but has the advantages of (a) computational simplicity and (b) applicability to all data, even where quantitative predictions would be expected to be systematically in error. The procedure was utilized to examine the degree to which an individual's retrieval order could be predicted from his rehearsal protocol.

For a given rehearsal protocol and "pool" of items retrieved, the first item retrieved was used as a starting

Table 4

Predicted and Observed Proportions of Items Retrieved on Draws 1 and 2 for Condition and Serial Position

\begin{tabular}{|c|c|c|c|c|c|c|c|c|c|c|c|c|c|c|c|}
\hline \multirow[b]{2}{*}{ Condition } & \multirow[b]{2}{*}{ Draw } & \multirow[b]{2}{*}{1} & \multirow[b]{2}{*}{1} & \multirow[b]{2}{*}{2} & \multirow[b]{2}{*}{3} & \multirow[b]{2}{*}{4} & \multirow[b]{2}{*}{5} & \multicolumn{3}{|c|}{ Serial Position } & \multirow[b]{2}{*}{9} & \multirow[b]{2}{*}{10} & \multirow[b]{2}{*}{11} & \multirow[b]{2}{*}{12} & \multirow[b]{2}{*}{13} \\
\hline & & & & & & & & & 7 & 8 & & & & & \\
\hline \multirow[t]{2}{*}{ RECALL } & 1 & Observed & .112 & .144 & .062 & .050 & .038 & .025 & .031 & .025 & .019 & .006 & .019 & .012 & .031 \\
\hline & & Predicted & .094 & .131 & .073 & .060 & .035 & .032 & .038 & .026 & .026 & .020 & .029 & .026 & .023 \\
\hline \multirow[t]{2}{*}{. } & 2 & Observed & .108 & .076 & .115 & .076 & .032 & .038 & .045 & .051 & .025 & .032 & .025 & .025 & .019 \\
\hline & & Predicted & .087 & .091 & .094 & .069 & .052 & .042 & .041 & .034 & .034 & .028 & .034 & .033 & .033 \\
\hline \multirow[t]{4}{*}{6} & 1 & Observed & .079 & .237 & .118 & .013 & .026 & & .026 & .026 & .013 & .026 & .013 & .000 & .053 \\
\hline & & Predicted & .116 & .167 & .107 & .063 & .050 & & .028 & .023 & .024 & .018 & .022 & .015 & .022 \\
\hline & 2 & Observed & .144 & .066 & .118 & .053 & .079 & & .013 & .053 & .053 & .000 & .039 & .039 & .026 \\
\hline & & Predicted & .098 & .114 & .116 & .074 & .060 & & .040 & .034 & .030 & .027 & .028 & .027 & .028 \\
\hline \multirow[t]{4}{*}{13} & 1 & Observed & .103 & .167 & .051 & .064 & .038 & .026 & .026 & .038 & .026 & .026 & .051 & .000 & \\
\hline & & Predicted & .090 & .123 & .068 & .057 & .043 & .033 & .032 & .042 & .027 & .033 & .031 & .028 & \\
\hline & 2 & Obseryed & .103 & .128 & .115 & .051 & .051 & .038 & .000 & .064 & .051 & .026 & .038 & .038 & \\
\hline & & Predicted & .089 & .097 & .087 & .062 & .052 & .043 & .041 & .041 & .038 & .032 & .038 & .035 & \\
\hline
\end{tabular}


point. From this point, the item most likely to be drawn was chosen from the pool, with this decision based on relative frequency of first-order transitions (higher orders considered only if necessary). The item chosen was then removed from the pool, made the starting point, and the same process repeated until the pool was exhausted.

In this way a "most likely path" through the items recalled was generated by the matrix of rehearsal transitions. In most cases, the procedure was quite simple, since individual rehearsal patterns were frequently quite stereotyped.

This algorithm was applied to each of the 320 subject-list protocols. In a few cases, an individual transition matrix was not regular, i.e., there was no possible path from one subset of items to another subset. In such cases, sets of "communicating items" were treated as individual protocols.

For each protocol, Kendall's $\tau$ coefficient was computed between the orders of the "most likely path" and the observed retrieval order. Over all protocols, the mean $\tau$ was +.494 . The ratio of positive to negative individual cases was approximately $9: 1$, with each of the 40 subjects showing a positive mean $\tau$ coefficient.

Final Recall. Immediate recall requires transitions between items originally presented in a single list. Final recall entails retrieval of items from separate lists, but no rule has been offered for dealing with transitions between items from separate lists. One contrivance to deal with this problem is the following: Assume that at the initiation of any sequence of same-list items during final recall there are no salient cues to cause deviations of the probability vector $\overrightarrow{\mathrm{R}}_{\mathbf{q}}$ from its equilibrium vector $\delta$. If $\delta$ does not vary systematically between lists, then the proportion of times that the item in serial position $\mathrm{j}$ is retrieved during any same-list sequence will not deviate systematically from $\delta_{j}$. Accordingly, the final recall serial position curve should be proportional to $\bar{\delta}$.

In order to test this line of speculation, final recall data was pooled over experimental conditions. The limiting vector $\delta$ for the pooled rehearsal probability matrix is plotted in Figure 4 (labeled Assumption A), as is the observed proportion of items in final recall from each serial position, based on 320 observations/point except for Positions 6 and 13, which are based on 240 . The two curves appear reasonably close over all serial positions, but there are reliable deviations, $\chi^{2}(19)=32.2, p<.05$.

It should be noted that the number of items recalled from particular lists was a monotonically decreasing function of the time since the list was shown, with frequencies of $88,111,131,131,158,181,217$, and 319 for Lists 2 through 9, respectively; this is consistent with findings discussed by Murdock (1972). Clearly, the final recall test is not measuring some permanent

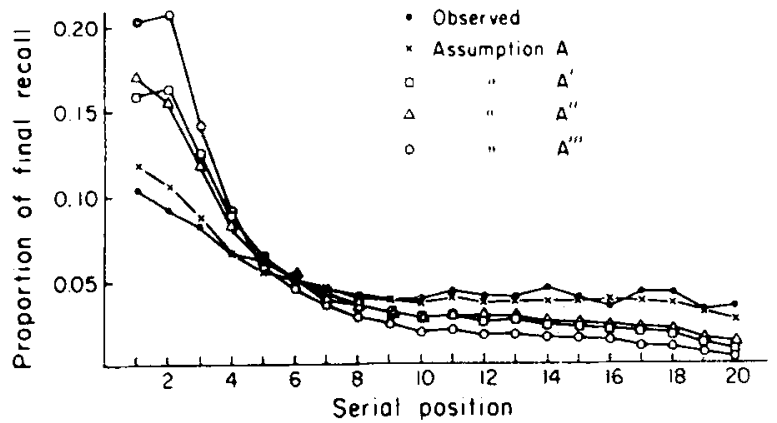

Figure 4. Limiting vectors, $\vec{\delta}$, for the model under Assumptions $\mathbf{A}, \mathbf{A}^{\prime}, \mathbf{A}^{\prime \prime}$, and $\mathbf{A}^{\prime \prime \prime}$ and comparable proportions observed.

strength which characterizes the long-term component of every list. It might, accordingly, have been more consistent with the above assumption to disregard data from the final list, which is more likely than other lists to have been influenced by transient cues. When this is done, one finds appreciably better agreement between $\bar{\delta}$ and the final recall curve, $\chi^{2}(19)=20.9, p>.20$.

Alternative Hypotheses. It is a natural extension of the present framework to consider alternative hypotheses about how encoding influences retention. Each of the following three hypotheses was independently tested against final recall data and against immediate recall data using Equation 3.

Hypothesis $A^{\prime}$ was identical to A except for the exclusion in tabulating transition frequencies of immediate, or "rote," repetitions during rehearsal. This hypothesis might be considered an extension of arguments made by Tulving (1968) for the irrelevance of rote repetitions in verbal recall and appears generally consistent with any model for verbal recall (e.g., Mandler, 1968) which takes the relevant memory structure to be that of a directed graph. Directed graphs do not contain "loops" from a node directly to itself. If Hypothesis $A^{\prime}$ led to more accurate predictions than Hypothesis $A$, it would have been interpreted as support for the irrelevance in verbal recall encoding of rote repetitions, i.e., transitions from an item's representation to itself.

Hypothesis $A^{\prime \prime}$ was identical to A except for exclusion of the first overt pronunciation of each item in determining frequency matrix $T$. This hypothesis seemed plausible, since the long-term status of an item read may differ from the status of one retrieved (Hogan \& Kintsch, 1971).

Hypothesis A"' was identical to A except that all overt rehearsals of an item less than $5 \mathrm{sec}$ after cessation of that item's presentation were ignored in evaluating $T$. Five seconds probably allows enough interfering rehearsals to lower the item's likelihood of being in primary memory. This way of evaluating $T$ should, 
therefore, be more sensitive to rehearsals involving secondary memory. Hypothesis A"' seemed consistent with results of Craik (1970) suggesting that retrieval from primary memory has little beneficial influence on long-term retention.

Hypothesis $\mathrm{A}$ and each alternative assumption yields a limiting vector presented in Figure 4. Comparisons show much better fit when every overt pronunciation is taken into account. Using the first pronunciation, "primary memory rehearsals," and/or all rote repetitions have a leveling influence on the limiting vector and presumably the structural component of serial position curves. This leveling is necessary to achieve adequate agreement between theory and final recall data.

Using the alternative hypotheses in conjunction with Equation 3 to predict immediate recall curves led to conclusions which are essentially identical. The leveling influence was quite evidence and is consistent with immediate recall results reported by Buschke and Kintsch (1970), Fischler, Rundus, and Atkinson (1970), and Glanzer and Meinzer (1967).

Hypothesis A's greater accuracy, in comparison to Hypotheses $A^{\prime \prime}$ and $A^{\prime \prime \prime}$, suggests that attention paid to an item appreciably increased its relative likelihood of long-term retention whether the item was merely read, rehearsed shortly after presentation, or rehearsed more than $5 \mathrm{sec}$ after presentation. The experiment was not designed to seek subtle differences in degree of effectiveness among these three types of processing.

The superiority of $A$ over $A^{\prime}$ indicates that transitions from an item to itself are, for some reason, important in determining verbal recall. According to the present model, the structure mirrored by patterns of rehearsal may be thought of as a network but does not have the form of a directed graph.

\section{GENERAL DISCUSSION}

The main point of the present theory and experiment is this: If one finds reasonable the original hypothesis about encoding and retrieval, then one should be prepared to expect serial position effects which follow from specific details of the encoding. If one does not accept the hypothesis or disputes some aspect of its present application, one should still be aware of potential contributions to serial position phenomena from related encoding and/or search processes.

The ideas presented in this paper should not be considered strictly with respect to their adequacy as a model for verbal recall. No attempt has been made here to deal exhaustively with problems or processes in verbal recall, and much more global models have been proposed (e.g., by Atkinson \& Shiffrin, 1968; Norman \& Rumelhart, 1970; or Shiffrin, 1970).

The intention was, rather, to develop a framework for dealing with encoding and search in verbal recall which might prove helpful in relating the memory mechanisms used in this task to those used in other cognitive tasks. With respect to this goal, there appear to be three general topics that merit discussion: the description of verbal recall mechanisms, the relationship of verbal recall to the development of cognitive structures, and possible sequential effects due to the utilization of a cognitive structure.

\section{Verbal Recall Mechanisms}

Models for verbal recall have not fully exploited the widely accepted principle that subjects develop and use cognitive structures, even to the extent of imposing subjective organization on nominally unrelated items. The hypothesis proposed here takes this insight as fundamental, relates details of subjective encoding to a specifiable cognitive structure, and allows serial position effects to emerge as consequences of retrieval operations on the cognitive structure.

Two results of the analysis have potentially important implications for mechanisms of verbal recall. First, the model offers an interitem encoding interpretation for various interesting results of Rundus, which would at a superficial level seem to call for an "item strength" explanation. Second, the model rationalizes the possible existence of a "transient" component of serial position curves which would ordinarily contribute to free recall recency effects but which is determined, not by recency, but by where memory search begins.

Experimental results reported in this paper tend to support the tenability of both ideas, but one experiment is obviously not "crucial." The conceptual possibility is more important than the test.

\section{Development of Cognitive Structures}

Cognitive theories are concerned with specifying details of cognitive structure and rules for its utilization in given tasks. By characterizing structures in terms of subjective operations of encoding rather than in terms of linguistic or algebraic theory, it might be possible to trace such structures through arbitrary levels of development; this has, of course, been a primary motivation for much research in verbal recall (see, e.g., Tulving, 1968).

\section{Sequential Effects Due to Utilization of a Cognitive Structure}

The result of interitem encoding, according to the present hypothesis, is a matrix $\mathrm{P}$ which operates on locations in a linear vector space. The elements of the matrix may be considered as indices of link strength in a network (Rips, Shoben, \& Smith, 1973). Viewed in this way, the present model is original in that (a) the links are labeled not by semantic relations but by experimentally defined relations of temporal contiguity and (b) the retrieval rule is appropriate for generating 
not just a single decision but a sequence of responses. With respect to Point a, the model gives a way of dealing with what Tulving (1968) has called "primary organization," i.e., consistent discrepancies between input and response orders independent of the subject's preexperimentally acquired semantic structures. With respect to Point $b$, rated or derived cognitive structures could be exploited, as easily as the measure of conjoint encoding frequency used here, to specify a regular "search choice matrix." It might, accordingly, be possible to deal with phenomena analogous to verbal recall serial position effects in tasks which are exercises of semantic memory.

\section{APPENDIX A} and

Let $\vec{\pi}=\vec{R}_{0}=\vec{R}_{0}^{\prime}=(0,1)$, i.e., $\pi_{1}=0$ and $\pi_{2}=1$,

$$
P=\left(\begin{array}{cc}
2 / 3 & 1 / 3 \\
1 / 3 & 2 / 3
\end{array}\right)
$$

Then

$$
\vec{R}_{1}=\pi \mathrm{T}=(0,1) \quad\left(\begin{array}{cc}
2 / 3 & 1 / 3 \\
1 / 3 & 2 / 3
\end{array}\right)=(1 / 3,2 / 3),
$$

i.e., $\quad R(1,1)=1 / 3$ and $R(2,1)=2 / 3$. Now, applying Equation 2 to Item 1 on Draw 1 ,

$$
\begin{aligned}
R(1,1)=1 / 3 & =R^{\prime}(1,1)+\underset{n=0}{1-1}\left(P^{1-n}\right)_{11} R^{\prime}(1 ; n) \\
1 / 3 & =R^{\prime}(1,1)+p_{11} R^{\prime}(1,0)=R^{\prime}(1,1)+p_{11} \pi_{1} \\
1 / 3 & =R^{\prime}(1,1)+2 / 3 \cdot 0 .
\end{aligned}
$$

$R^{\prime}(1,1)=1 / 3$, i.e., the item in Serial Position 1 has probability $1 / 3$ of appearing for the first time on the first draw and probability $R(1,1)-R^{\prime}(1,1)=1 / 3-1 / 3$ $=0$ of appearing for the second time on the first draw. Applying Equation 2 to Item 2 on Draw 1,

$$
\begin{aligned}
R(2,1)=2 / 3 & =R^{\prime}(2,1)+\sum_{n=0}^{1-1}\left(P^{1-n}\right)_{22} R^{\prime}(2, n) \\
2 / 3 & =R^{\prime}(2,1)+p_{22} R^{\prime}(2,0)=R^{\prime}(2,1)+p_{22} \pi_{2} \\
2 / 3 & =R^{\prime}(2,1)+2 / 3 \cdot 1 .
\end{aligned}
$$

$R^{\prime}(2,1)=0$, i.e., the item in Serial Position 2 has probability 0 of appearing for the first time on the first draw and probability $R(2,1)-R^{\prime}(2,1)=2 / 3-0=2 / 3$ of appearing for the second time on the first draw. The serial position curves on Draw 1 are $(1 / 3,0)$ for "first recall" and $(0,2 / 3)$ for "repetition."

\section{APPENDIX B}

Assume that the matrix $P$ is nonsingular, i.e., $\operatorname{det}(P) \neq 0$. Let $X_{j}={ }_{j=1}^{n} t_{i j}=$ the number of times $a$ transition was made to $11 \mathrm{em} j$ or the $\mathrm{j}^{\text {th }}$ column sum of T. Let $Y_{i}=\sum_{i=1}^{n} t_{i j}=$ the number of times a transition was made from item $i$ or the $i^{\text {th }}$ row sum of $T$. We know

$$
t_{i j}=Y_{i j} p_{i j}
$$

If the vector $\vec{X}$ is proportional to the limiting vector $\bar{\delta}$ of $\mathrm{P}$, then

$$
\overrightarrow{\mathrm{X}} p=\overrightarrow{\mathrm{X}}
$$

From Equation B !,

$$
X_{j}=\sum_{i=1}^{n} t_{i j}=\sum_{i=1}^{n} Y_{i} p_{i j}=(\bar{Y} P)_{j}
$$

or

$$
\vec{X}=\vec{Y} P \text {. }
$$

Thus,

$$
\vec{X} P=\vec{Y} P .
$$

Since $P$ is nonsingular, it possesses an inverse, $P^{-1}$. Then

$$
\begin{aligned}
\vec{X} P^{P} P^{-1} & =\vec{Y} P P^{-1} \\
\vec{X} & =\vec{Y} .
\end{aligned}
$$

Thus, $\overrightarrow{\mathrm{X}}$ is proportional to $\bar{\delta}$ implies that the column sums of $T$ equal the row sums of $T$.

Conversely, if

$$
\overrightarrow{\mathrm{Y}}=\overrightarrow{\mathrm{X}}
$$

then by Equation $\mathrm{B} 3$

$$
\vec{X}=\vec{Y} P
$$

so

$$
\vec{X}=\vec{X} P
$$

and $\overrightarrow{\mathrm{X}}$ is proportion to $\vec{\delta}$.

Accordingly, the vector $\delta$ is proportional to the vector $\vec{X}$ of column sums of $T$ if, and only if, the column sums of $T$ equal the corresponding row sums of $\mathrm{T}$. This was approximately true of the rehearsal data reported in Table 1.

\section{REFERENCES}

Atkinson, R. C., \& Shiffrin, R. M. Human memory: A proposed system and its control processes. In $K$. W. Spence and J. T. Spence (Eds.), Advances in the psychology of learning and Spence (Eds.), Advances in the psychology of learning and
motivation: Research and theory. Vol. 2. New York: Academic Press, 1968.

Bousfield, A. K. Whitmarsh, G. A., \& Esterson, J. Serial position effects and the Marbe effect in free recall of meaningful words. Journal of General Psychology, 1958, 59, 255-262.

Bruce, D.. \& Papay. J. P. Primacy effect in single trial tree recall. Journal of Verbal Learning and Verbal Behavior, 1970,9, 473-486.

Buschke, H., \& Kintsch, W. Rehearsal strategies and the serial-position curve in immediate free recall of ordered items. Quarterly Joumal of Experimental Psychology, 1970, 2, 347-352. 
Cohen, R. L. Recency effects in long-term recall and recognition. Journal of Verbal Learning and Verbal Behavior, 1970, 9, 672-678.

Craik, F. I. M. Modality effects in short-term storage. Journal of Verbal Learning and Verbal Behavior, 1969, 8, 658-664.

Craik, F. I. M. The fate of primary memory items in free recall. Journal of Verbal Learning and Verbal Behavior, 1970, 9 143-148.

Crowder, $R$. G. The role of one's own voice in immediate memory. Cognitive Psychology, 1970, 1, 157-178.

Deese, J., \& Kaufman, R. A. Serial effects in recall of unorganized and sequentially organized verbal material. Journal of Experimental Psychology, 1957, 54, 180-187.

Fischler, I., Rundus, D., \& Atkinson, R. C. Effects of overt rehearsal processes on free recall. Psychonomic Science, 1970 $19,249-250$

Glanzer, M., \& Meinzer, A. The effects of intralist activity on free recall. Journal of Verbal Learning and Verbal Behavior, $1967,6,929-935$.

Hogan, R. M., \& Kintsch, W. Differential effects of study and test trials on long-term recognition and recall. Journal of Verbal Learning and Verbal Behavior, 1971, 10, 562-567.

Kemeny, J. G., \& Snell, J. L. Finite Markov chains. Princeton, N.J: Van Nostrand, 1961.

Kintsch, W., Miller, R. F., \& Hogan, R. M. Semantic structure and clustering in free recall. Technical Report, University of Colorado, 1970 .

Mandler, G. Association and organization: Facts, fancies, and theories. In T. R. Dixon and D. L. Horton (Eds.), Verba behavior and general behavior theory. Englewood Cliffs, N,J: Prentice-Hall, 1968.

Meyer, D. E., Schvaneveldt, R. W., \& Ruddy, M. G. Activation of lexical memory. Paper presented at the meeting of the Psychonomic Society, St. Louis, Missouri, November 1972.

Murdock, B. B., Jr. Short-term memory. In G. H. Bow er (Ed.) The psychology of learning and motivation: Aduances in research and theory. Vol. 5. New York: Academic Press, 1972 .
Norman, D. A., \& Rumelhart, D. E. A system for perception and memory. In D. A. Norman (Ed.). Models of human memory. New York: Academic Press, 1970.

Rips, L. J., Shoben, E. J., \& Smith, E. E. Semantic distance and the verification of semantic relations. Journal of Verbal Learning and Verbal Behavior, 1973, 12, 1-20.

Rumelhart, D. E. \& Abrahamson, A. A. A model for analogical reasoning. Cognitive Psychology, 1973, 5, 1-28.

Rundus, D. Analysis of rehearsal processes in free recall. Journal of Experimental Psychology, 1971, 89, 63-77.

Shiffin, R. M. Memory search. In D. A. Norman (Ed.), Models of human memory. New York: Academic Press, 1970.

Tulving, E. The effects of presentation and recall of material in free recall learning, Journal of Verbal Learning and Verbal Behavior, $1967,6,175-184$.

Tulving, E. Theoretical issues in free recall. In T. R. Dixon and D. L Horton (Eds.), Verbal behavior and general behavior theory. Englewood Cliffs, N.J: Prentice-Hall, 1968.

Waugh, N. C., \& Norman, D. A. Primary memory. Psychological Review, 1965, 72, 89-104

\section{NOTES}

1. The arguments which follow were developed with the overt rehearsal experiment in mind. To the extent that the method of overt rehearsal does not accurately reflect processes of encoding. what follows still constitutes a way of dealing with interitem encoding and its ramifications, but the means of evaluating the encoded structure must be more indirect. The primary concern will be developing interesting relationships which might follow from properties of the encoded structure.

2. Although there were 74 relevant predictions, four cells included no correct responses; for these cells the relevant contribution to $x^{2}$ was undefined.

(Received for publication March 8, 1974; revision received July $29,1974$. ) 